\title{
Editorial Publication Matters
}

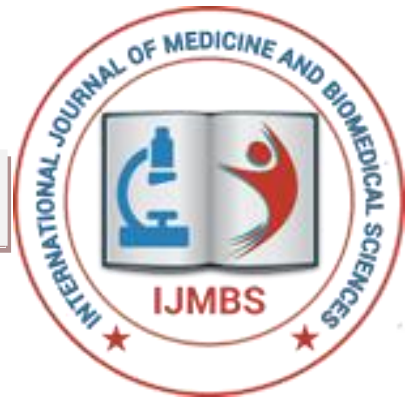

Kiran Sapkota

Ph.D. Scholars- University of Iowa, USA

International Journal of Medicine and Biomedical Sciences (IJMBS) is a platform for researchers to publish their research across various disciplines. The journal has been initiated by academicians who specialize in different fields such as cell biology, biochemistry, medical chemistry, medical science, microbiology and public health to name but a few. The primary aim of the journal is to publish original research to enhance our current knowledge of medical, biomedical and public health sciences.

A researcher spends a significant time in doing research. After the completion of the study, it is the time for the publication of the hard work. However, many researchers are reluctant to write and publish the research paper once the project is completed. The famous saying "publish or perish" is true because timely publication of research paper is essential to progress in their carriers.

With the aim of promoting both students and scholars' research, we have made a publication process much easier and faster. That is to say, a researcher will upload the final draft of the research paper. Once the research paper is received, it will be sent to the reviewers for feedback and evaluation. The article will be accepted, returned for revision or rejected. All these process are managed through the online system to expedite the review and publication process.
As a publication of a research article concludes the research process, we invite scholars and researchers to submit original research articles, reviews, commentaries and case studies. Our experts review board panel will, and the editorial board will be available to answer any queries you have. Additionally, feel free to explore the webpage of the journal to know about the submission process, requirement for publication and get updates on your submission. 